\title{
Radiative accidental matter
}

\section{Aristizabal Sierra, ${ }^{a, b}$ C. Simoes ${ }^{a}$ and D. Wegman ${ }^{a}$}

${ }^{a}$ IFPA, Dép. AGO, Université de Liège,

Bât B5, Sart Tilman B-4000 Liège 1, Belgium

${ }^{b}$ Universidad Técnica Federico Santa María - Departamento de Física, Casilla 110-V, Avda. España 1680, Valparaíso, Chile

E-mail: daristizabal@ulg.ac.be, csimoes@ulg.ac.be, dwegman@ulg.ac.be

ABSTRACT: Accidental matter models are scenarios where the beyond-the-standard model physics preserves all the standard model accidental and approximate symmetries up to a cutoff scale related with lepton number violation. We study such scenarios assuming that the new physics plays an active role in neutrino mass generation, and show that this unavoidably leads to radiatively induced neutrino masses. We systematically classify all possible models and determine their viability by studying electroweak precision data, big bang nucleosynthesis and electroweak perturbativity, finding that the latter places the most stringent constraints on the mass spectra. These results allow the identification of minimal radiative accidental matter models for which perturbativity is lost at high scales. We calculate radiative charged-lepton flavor violating processes in these setups, and show that $\mu \rightarrow e \gamma$ has a rate well within MEG sensitivity provided the lepton-number violating scale is at or below $5 \times 10^{5} \mathrm{GeV}$, a value (naturally) assured by the radiative suppression mechanism. Sizeable $\tau \rightarrow \mu \gamma$ branching fractions within SuperKEKB sensitivity are possible for lower lepton-number breaking scales. We thus point out that these scenarios can be tested not only in direct searches but also in lepton flavor-violating experiments.

Keywords: Beyond Standard Model, Neutrino Physics

ARXIV EPRINT: 1605.08267 


\section{Contents}

1 Introduction 1

2 Effective scales in accidental matter scenarios 3

2.1 Accidental matter representations 5

3 Higher-order SU(2) representations and their cosmological instability 7

4 Radiative accidental matter $\quad 8$

$\begin{array}{ll}4.1 & \text { Additional constraints: } \rho \text { parameter and BBN }\end{array}$

$\begin{array}{lll}4.2 \text { UV completions and perturbativity } & 12\end{array}$

$\begin{array}{lll}4.3 & \text { Lepton flavor violation: generic approach } & 14\end{array}$

5 Conclusions 18

A Two-loop renormalization group equations $\quad 19$

\section{Introduction}

Various theoretical and experimental arguments support the idea that at certain energy scale new degrees of freedom should be operative. A solution to the electroweak (EW) hierarchy problem demands this scale to be order $\mathrm{TeV}$ and leads to new physics potentially testable at the LHC. This new physics is expected to address not only the hierarchy problem, but to account as well for experimental-driven puzzles such as the origin of dark matter [1], neutrino masses [2-4] and the baryon asymmetry of the universe [1]. Typical models follow a rather simple approach in which sectors subject to strong phenomenological constraints are decoupled, while those for which experimental bounds (of whatever nature) are somewhat weaker are associated with low-energy scales.

This is the case for lepton number-violating (LNV) physics, which in the absence of a "non-conventional" suppression mechanism ${ }^{1}$ demands GUT-scale physics. If one adopts that approach, the smallness of neutrino masses "naturally" arise due to the presence of decoupled states that can be related as well with a solution to the baryon asymmetry problem (see e.g. [5-9]) and that allows for embeddings of such scenarios in GUTs. In such picture the LNV physics, apart from accounting for low-energy neutrino observables, does not leave any experimental trace, something consistent with direct and indirect experimental searches, see e.g. [10-12]. The TeV sector that accounts for the hierarchy problem and that might involve dark matter (DM) states, contribute to various rare processes whose

\footnotetext{
${ }^{1}$ By non-conventional we refer to mechanisms where the suppression does not rely on the presence of GUT-scale states, i.e. radiative or slightly broken lepton number mechanisms, see section 2.1.
} 
current bounds are as well tight $[13,14]$. Consistency therefore requires a mechanism that allows for $\mathrm{TeV}$ states while explaining the absence of signals in the high-intensity frontier.

A rather popular approach to such problem is given by the minimal flavor violation hypothesis, that postulates that the only source of flavor violation are the standard model (SM) Yukawa couplings, regardless of the nature of the new physics [15]. In the quark sector this assumption automatically reconciles $\mathrm{TeV}$-scale physics with the non-observation of flavor-violating signals in indirect searches. In the lepton sector despite not being univocally implementable it leads to a consistent picture that in its minimal realizations entails quite a few predictions for lepton flavor-violating processes [16]. Accidental matter, is another interesting approach that although does not aim at addressing the EW hierarchy problem it enables for new order-TeV physics without invoking any special flavor structure [17]. The idea is that the new physics should preserve all the SM exact and approximate symmetries up to a cutoff scale $\Lambda$, above which these symmetries are presumably broken by a larger theory. Assuming that this cutoff scale is universal and determined by the constraints implied by lepton number violation (in the absence of a "non-conventional" suppression mechanism), a simple but compelling minimal picture emerges with new order-TeV states that do not conflict with negative results from indirect experimental searches.

In this paper we define and study alternative forms of accidental matter models which we dub radiative accidental matter. ${ }^{2}$ For that aim we allow for a mismatch among the different scales involved: LNV, quark- and lepton-flavor violating (QFV and LFV), baryonnumber violating (BNV) and the scale at which perturbativity is lost. Furthermore, we show that if the accidental matter representation plays an active role in neutrino mass generation this unavoidably leads to radiative neutrino masses (hence the name radiative accidental matter), thus providing a "natural" suppression mechanism that enables for low-scale lepton number violation, which reopens the possibility of potentially large LFV effects, thus increasing the testability of these scenarios.

The full set of accidental matter representations are derived from the condition that the new degrees of freedom preserve the SM accidental and approximate symmetries up to a certain cutoff scale. This set contains lower- and higher-order SU(2) representations, with the latter in some cases defining minimal DM models [19-21]. This however is not the case in the scenarios we will discuss. As has been recently pointed out in refs. [22, 23], once a lower cutoff scale is allowed (in this case related with neutrino physics constraints) the neutral component contained in the representation decays fast, with typical lifetimes amounting to $\mu \mathrm{s}$. This observation combined with a lower perturbative scale enables nonvanishing hypercharge sextets, that otherwise would be forbidden [17]. Moreover, in our analysis we will not consider hypercharge-zero septets since their quartic scalar couplings reach Landau poles at rather low scales, $\sim 10^{7} \mathrm{GeV}[24]$.

The rest of this paper is organized as follows. In section 2.1 we discuss the different energy scales of the problem, define a benchmark scenario for radiative accidental matter and determine the set of relevant representations. In section 3 we present our arguments

\footnotetext{
${ }^{2} \mathrm{~A}$ systematic classification of $\mathrm{U}(1)_{B-L}$ loop-induced neutrino mass models has been presented in ref. [18].
} 
for cosmological instability of higher-order $\mathrm{SU}(2)$ representations, paying special attention to the scalar sextet. In section 4.1 various phenomenological constraints are discussed, while in section 4.2 we examine the different UV completed radiative accidental matter models and study their perturbative behavior. In section 4.3 we study in a fairly modelindependent way LFV processes in these scenarios. Finally, in section 5 we summarize and present our conclusions. In appendix A we present the two-loop renormalization group equations (RGEs) that we have used in our analysis.

\section{Effective scales in accidental matter scenarios}

Accidental matter models are weak-scale extensions of the SM in which the beyond-SM (BSM) degrees of freedom $(\boldsymbol{R})$ preserve the accidental and approximate symmetries of the $\mathrm{SM}$ at the renormalizable level. Thus, this means that even if present at the renormalizable level their effects will not be accessible in indirect searches. One might wonder whether departures from their standard formulation could change that picture. For that aim one can consider the SM as the renormalizable part of a larger Lagrangian:

$$
\mathcal{L}=\mathcal{L}_{\mathrm{SM}}^{\mathrm{Ren}}+\sum_{N>4} \frac{\mathcal{C}_{N}}{\Lambda_{\mathrm{Eff}}^{N-4}} \mathcal{O}_{N}
$$

where $\mathcal{L}_{\mathrm{SM}}^{\mathrm{Ren}}$ are the renormalizable SM interactions, whereas the second term are effective operators where the only dynamical degrees of freedom are SM fields. They do break the SM accidental and approximate symmetries and so their effects include CP and flavor violation in the quark and lepton sectors as well as baryon and lepton number breaking.

In the standard approach the coefficients of the effective expansion $\mathcal{C}_{N}$ are assumed to be $\mathcal{O}(1)$. When combined with the assumption that neutrinos are Majorana particles, this fixes the effective scale, $\Lambda_{\mathrm{Eff}} \sim 10^{15} \mathrm{GeV}$. Automatically then, all possible signatures related with departures from SM accidental and approximate symmetries are suppressed, thus explaining their absence in indirect searches. Testability of these scenarios is possible only if the new states can be directly produced and detected in collider experiments. Otherwise the new physics, although present, could be hard - if not impossible - to reveal. A possible departure from the standard formulation consists then in allowing for lower cutoff scales that in turn allow for other observables to have sizeable values, thus increasing the testability of these scenarios.

The effective operators in (2.1) are subject to different phenomenological constraints, with the most stringent limits enforced by neutrino masses on the LNV ones. Without any further assumption the leading-order LNV operator is given by the dimension five Weinberg operator

$$
\frac{\mathcal{C}_{i j}}{\Lambda}\left(\overline{\ell_{i}^{c}} i \tau_{2} H\right)\left(H^{T} i \tau_{2} \ell_{j}\right)
$$

that after EW symmetry breaking leads to $m_{\nu} \sim \mathcal{C} v^{2} / \Lambda$. A lower cutoff scale, say $\mathcal{O}(\mathrm{TeV})$, is possible provided $\mathcal{C} \sim 10^{-12}-10^{-11}$. There are two generic mechanisms through which such a small coefficient can be naturally obtained: (i) the operator is related with a slightly broken symmetry (for example slightly broken lepton number) [25-28], (ii) the operator is 


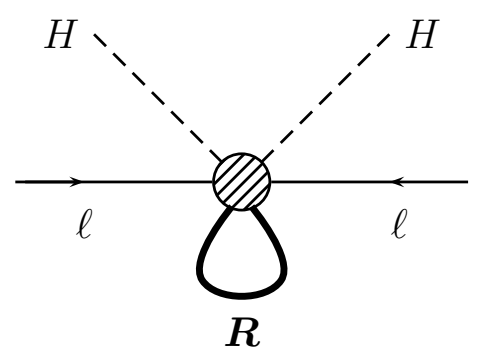

Figure 1. Dimension five LNV effective operator in the limit SM $+\boldsymbol{R}$. The presence of $\boldsymbol{R}$ allows for a naturally small expansion coefficient $\mathcal{C}$ and therefore for a lower effective scale.

radiatively induced. Though mechanisms of type (i) can be envisaged, in the presence of $\mathrm{SM}+\boldsymbol{R}$ the natural option relies on (ii). Let us discuss this in more detail. Below $\Lambda$ the only degrees of freedom are the SM fields and $\boldsymbol{R}$, and so lepton number violation should be determined by effective operators (as required by the accidental matter scenario). Since $\boldsymbol{R} \otimes \boldsymbol{R} \supset \mathbf{1} \oplus \mathbf{3} \oplus \mathbf{5} \oplus \cdots \oplus(2 \boldsymbol{R}-1)$, the effective operator in (2.2) can be endowed with the new degree of freedom as shown in figure 1. Therefore, in this case the effective expansion coefficient is suppressed by the loop factor and by extra couplings that originate in the UV completed theory. Roughly it can be written as $\mathcal{C} \sim Y_{\nu}^{4} / 16 \pi^{2}$, which means that $\Lambda$ can be as small as $\sim 10^{5} \mathrm{GeV}$ for $Y_{\nu} \sim h_{\tau}$ ( $\tau$ Yukawa coupling). These values mean that the degrees of freedom of the UV theory, although not reachable at the LHC can manifest in indirect searches, e.g. in $\mu \rightarrow e \gamma, \mu \rightarrow 3 e$ or in $\mu-e$ conversion in nuclei, processes that are/will be searched for in MEG [12], Mu3e [29, 30] and PRISM/PRIME [31, 32] respectively (see e.g. refs. [27, 33] for phenomenological studies).

Some words are in order regarding the effective scale for the operator in (2.2). This scale determines the cutoff scale where different UV completions - involving new states - enable writing down the operator in figure 1 through renormalizable couplings. This scale differs from that where perturbativity is lost $\left(\Lambda_{\text {Landau-pole }}\right)$, in contrast to standard accidental matter scenarios where these two scales match (see figure 2). At scales above $\Lambda$ new states kick in (generically denoted by $\boldsymbol{R}^{\prime}$ ), and their renormalizable interactions break lepton number and lepton flavor, but quark flavor and baryon number are still symmetries of the renormalizable Lagrangian (at that scale). Since the new states contribute to $\alpha_{1}$ and $\alpha_{2}\left(\alpha_{i}=g_{i}^{2} / 4 \pi\right)$ running, perturbativity is lost more rapidly than in the case $\mathrm{SM}+\boldsymbol{R}$. The exact value where this happens depends upon the number and dimensionality of the new representations. Assuming that perturbativity is restored by a larger gauge theory, one could expect quark flavor and/or baryon number to be broken at that scale. If only quark flavor is broken, $\Lambda_{\text {Landau-pole }} \sim 10^{8} \mathrm{GeV}$ suffices to satisfy current constraints on QFV processes [34], otherwise $\Lambda_{\text {Landau-pole }} \gtrsim 10^{15} \mathrm{GeV}$ is required to ensure proton stability. Here we will select viable accidental matter scenarios by the condition $\Lambda_{\text {Landau-pole }} \gtrsim 10^{8} \mathrm{GeV}$, which implicitly assumes that the new dynamics does not involve any baryon number violation. 


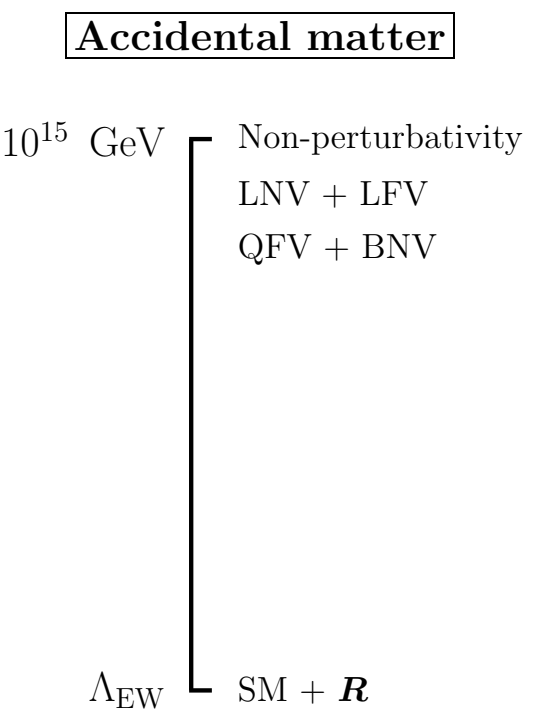

\section{Radiative accidental matter}

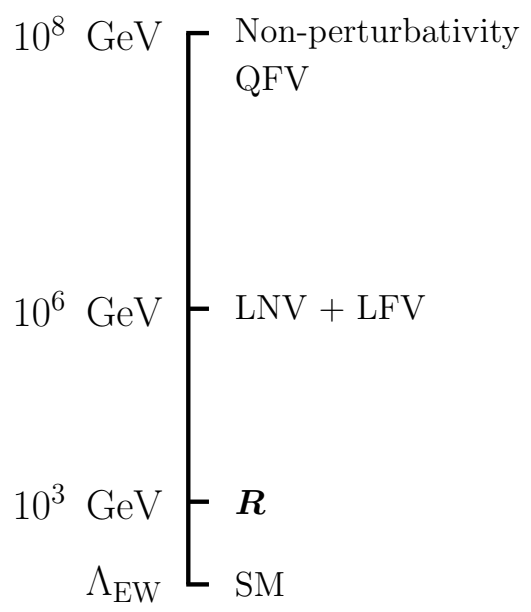

Figure 2. Energy scales in standard (left-hand side) and radiative (right-hand side) accidental matter models (benchmark scenario). Note that the LNV, LFV, and QFV scales in the latter are smaller, thus allowing for potential observability of the corresponding processes. The energy scales on the right side represent the benchmark scenario we will use for our discussion.

\subsection{Accidental matter representations}

The quantum numbers of the new representation are determined by whether $\boldsymbol{R}$ is a fermion or a scalar. Since $\boldsymbol{R}$ should preserve the SM symmetries, as pointed out in [17] fermionic representations should be such that operators of the form

$$
\mathcal{O}_{N=4} \sim \boldsymbol{R} \mathcal{O}_{\mathrm{SM}}
$$

should not be possible writing. Otherwise new $\mathcal{G}_{F}=\mathrm{U}(3)_{Q} \otimes \mathrm{U}(3)_{d} \otimes \mathrm{U}(3)_{u} \otimes \mathrm{U}(3)_{\ell} \otimes \mathrm{U}(3)_{e^{-}}$ breaking sources will be introduced. For scalar representations instead renormalizable operators are possible without affecting $\mathcal{G}_{F}$, provided their quantum numbers do not enable couplings with SM fermionic bilinears. Bearing in mind this discussion and that

$$
\underbrace{\mathbf{2} \otimes \cdots \otimes \mathbf{2}}_{(R-1) \text { times }} \supset \boldsymbol{R}
$$

the first relevant fermionic representation is $\boldsymbol{R}_{F}=\mathbf{4}_{F}^{Y}$, with hypercharge $Y=1 / 2$ or $3 / 2$ determined by whether it couples to $\ell H H^{\dagger}$ or $\ell H^{\dagger} H^{\dagger}$ (here we will use the notation $\boldsymbol{R}_{F, S}^{Y}$ where $\boldsymbol{R}$ labels the representation, $F$ and $S$ its fermionic or scalar character and $Y$ its hypercharge. Hypercharge is normalized according to $\left.Q=T_{3}+Y\right)$. The remaining fermionic representations follow from the rule in (2.4) with hypercharge fixed by the SM operator to which they couple. For scalars, at the renormalizable level, the first representation is indeed a SM singlet, $\boldsymbol{R}_{S}=\mathbf{1}_{S}^{0}$, with the remaining representations given by $\boldsymbol{R}_{S}=\mathbf{3}_{S}^{0}, \mathbf{4}_{S}^{1 / 2}$, $\mathbf{4}_{S}^{3 / 2}$ and $\mathbf{6}_{S}^{1 / 2}$, with the latter being loop-induced (see section 3). ${ }^{3}$ For non-renormalizable

\footnotetext{
${ }^{3}$ Note that $\mathrm{SU}(2) \otimes \mathrm{U}(1)_{Y}$ invariance allows for $\boldsymbol{R}_{S}=\mathbf{3}_{S}^{1}$. This representation however couples to the fermion bilinear $\overline{\ell^{c}} \ell$ and so introduces $\mathcal{G}_{F}$-breaking sources.
} 


\begin{tabular}{|c|c|c|c|c|c|c|c|c|c|}
\hline \multicolumn{11}{|c|}{ Radiative accidental matter representations } \\
\hline$Y$ & $\mathbf{1}_{S}^{Y}$ & $\mathbf{2}_{S}^{Y}$ & $\mathbf{3}_{S}^{Y}$ & $\mathbf{4}_{S}^{Y}$ & $\mathbf{5}_{S}^{Y}$ & $\mathbf{6}_{S}^{Y}$ & $\mathbf{7}_{S}^{Y}$ & $\mathbf{4}_{F}^{Y}$ & $\mathbf{5}_{F}^{Y}$ \\
\hline 0 & $\checkmark$ & - & $\checkmark$ & - & $\checkmark$ & - & $\times$ & - & $\checkmark$ \\
\hline $1 / 2$ & - & - & - & $\checkmark$ & - & $\checkmark$ & - & $\checkmark$ & - \\
\hline 1 & - & - & - & - & $\checkmark$ & - & - & - & - \\
\hline $3 / 2$ & - & $\checkmark$ & - & $\checkmark$ & - & $\checkmark$ & - & $\checkmark$ & - \\
\hline 2 & - & - & - & - & $\checkmark$ & - & - & - & - \\
\hline $5 / 2$ & - & $\checkmark$ & - & - & - & $\checkmark$ & - & - & - \\
\hline
\end{tabular}

Table 1. Accidental matter representations obtained under the assumption that $\boldsymbol{R}$ plays an active role in neutrino mass generation. Checkmarks (dashes) indicate representations which are (not) viable. Blueish cells refer to representations involving renormalizable couplings. Reddish (greenish) cells refer instead to representations involving $\operatorname{dim}=5(\mathrm{dim}=6)$ non-renormalizable couplings. The list differs with what was found in standard accidental matter scenarios where lepton number violation occurs at $10^{15} \mathrm{GeV}[17]$ in that it contains scalar sextets and the septet is not allowed by perturbative criteria.

operators the first scalar representation is $\boldsymbol{R}_{S}=\mathbf{2}_{S}^{Y}$ with $Y=3 / 2,5 / 2$. The remaining scalar representations are: $\mathbf{5}_{S}^{Y}(Y=0,1,2), \mathbf{6}_{S}^{Y}(Y=3 / 2,5 / 2), \mathbf{7}_{S}^{Y}(Y=0,1,2,3), \mathbf{8}_{S}^{Y}$ $(Y=1 / 2,3 / 2,5 / 2,7 / 2)$ and so on. From this list, viable representations are selected from cosmological constraints and the condition of perturbativity.

The neutral component of higher-order $\mathrm{SU}(2)$ representations is cosmologically stable: for scalars $\boldsymbol{R}_{S}^{Y}>\mathbf{5}_{S}^{Y}$, while for fermions $\boldsymbol{R}_{F}^{Y} \geq \mathbf{5}_{F}^{Y}$. Direct DM searches constraints, however, rule out all those representations for which $Y \neq 0$ [19-21]. Perturbativity criteria as well places constraints on viable representations. Using a two-loop RGE analysis, ref. [17] has shown that for $\boldsymbol{R}_{S}^{0}>\mathbf{8}_{S}^{0}$ and $\boldsymbol{R}_{F}^{0}>\mathbf{6}_{F}^{0}$ a Landau pole is obtained for scales below $\sim 10^{8} \mathrm{GeV}$. Thus, in a model defined by $\mathrm{SM}+\boldsymbol{R}$ the accidental matter representations are: $\boldsymbol{R}_{S}^{Y} \leq \mathbf{7}_{S}^{0}$ and $\boldsymbol{R}_{F}^{Y}<\mathbf{5}_{F}^{0}$.

A major difference between the standard accidental matter models and the setups we are considering here is that in the latter there are new degrees of freedom that enter at relatively low scales $\left(10^{6} \mathrm{GeV}\right)$. The presence of these states induces fast decays of those representations that otherwise would be cosmologically stable [22]. Thus they no longer involve a DM particle, and therefore direct DM constraints no longer hold. This enables $Y \neq 0$ representations, something that is particularly important for the scalar sextet (see section 3). ${ }^{4}$

In summary, the setups we will consider henceforth are defined by the accidental matter representations listed in table 1 . This list differs from that found in standard accidental matter scenarios in that it contains the scalar sextet representations, which are enabled due to the instability of higher-order representations induced by the presence of additional

\footnotetext{
${ }^{4}$ This argument applies as well to $\mathbf{5}_{F}^{1,2}$, however for these representations alone a Landau pole is reached at scales below $\sim 10^{8} \mathrm{GeV}$. The presence of additional representations at $10^{6} \mathrm{GeV}$ reduces that scale to values well below those that define our perturbativity criteria (see figure 2), and so we do not consider them.
} 
representations. The UV completed models we will construct are therefore defined by these representations and subject to the condition of the full UV model satisfying $\Lambda_{\text {Landau-pole }}>$ $10^{8} \mathrm{GeV}$ (see section 4.2).

\section{Higher-order $\mathrm{SU}(2)$ representations and their cosmological instability}

As we have already pointed out, higher-order $\mathrm{SU}(2)$ representations in $\mathrm{SM}+\boldsymbol{R}$ models are cosmologically stable. The neutral component of the hypercharge-zero fermion quintet and scalar septet are - in principle - WIMP DM particles. For these representations tree level effective operators of the form (operators that induce DM decay)

$$
\mathcal{O}_{N}=\boldsymbol{R} \mathcal{O}_{\mathrm{SM}}
$$

with $\mathcal{O}_{\mathrm{SM}}$ an operator entirely consisting of only SM fields, are $\operatorname{dim}=6$ and $\operatorname{dim}=7$, respectively. Thus, lifetimes amounting to $10^{26}$ seconds (as required by the non-observation of $\gamma$-ray, $\nu, e^{+}$or $p^{-}$signals in DM indirect detection experiments [35-38]) are found for $\Lambda \gtrsim 10^{15} \mathrm{GeV}$ for $\mathbf{5}_{F}^{0}$ and $\Lambda \gtrsim 10^{10} \mathrm{GeV}$ for $\boldsymbol{7}_{S}^{0}$, provided $m_{\mathrm{DM}} \subset[5,10] \mathrm{TeV}$ [22]. However, if one considers one-loop induced effective operators one finds that scalar septet decays are instead driven by $\operatorname{dim}=5$ operators $[17,39]$, for which not even $\Lambda=M_{\text {Planck }}$ leads to sufficiently large DM decay lifetimes. This observation then singles out the hypercharge-zero fermion quintet as the only representation containing a viable DM particle.

It is worth pointing out that this representation is however subject to stringent constraints coming from indirect DM searches. Particularly relevant are limits derived from $\gamma$-ray line searches from the galactic center, for which it has been found that if the Milky Way exhibits a Navarro-Frenk-White or Einasto DM profile this representation is not viable either [40,41]. It can be however consistently considered in the context of cored profiles such as Burket or Isothermal. Or by relaxing the hypothesis of WIMP DM, allowing for $Y=\epsilon \ll 1$ and so leading to millicharged DM scenarios [39].

In scenarios defined by UV completions of the operator in figure 1, slow decays of higher-order EW representations do not hold anymore. The point is that at $\Lambda=10^{6} \mathrm{GeV}$ new states kick in, introducing new renormalizable couplings that allow writing down operators of type (3.1) with cutoff scales fixed by neutrino data. Roughly one can write $m_{\nu} \sim v^{2} Y_{\nu}^{4} / 16 \pi^{2} \Lambda$, which means that for $m_{\nu}=m_{\mathrm{Atm}}=50 \mathrm{meV}[42-44]$ and $Y_{\nu}=1$ the cutoff scale should be below $\sim 10^{13} \mathrm{GeV}$. This scale is below the value required for cosmological stability of the fermion quintet, thus showing that these setups are not reconcilable with slow DM decays (for a more detailed discussion see $[22,23]$ ). This can be put more precisely in the context of explicitly broken symmetries: DM slow decays can be understood as due to an accidental $\mathbb{Z}_{2}$ symmetry under which $\boldsymbol{R} \rightarrow-\boldsymbol{R}$ and $X_{\mathrm{SM}} \rightarrow X_{\mathrm{SM}}$, and which results as a consequence of the SM gauge symmetry. For $\boldsymbol{R}=\mathbf{5}_{F}^{0}$, UV completions of the operator in figure 2 always allow for couplings that break $\mathbb{Z}_{2}$, hence DM instability. ${ }^{5}$

\footnotetext{
${ }^{5}$ Sufficiently small $\mathbb{Z}_{2}$-breaking couplings can lead to cosmological stability of the neutral component of the multiplet and so can be used to reconcile minimal DM with loop-induced neutrino masses, see ref. [45] for more details.
} 
These arguments can apply to $Y \neq 0$ representations, depending on the value of $Y$ and on the specific UV completion. Indeed, they are responsible for the scalar sextet as a viable accidental matter representation as we now discuss. For $\mathbf{6}_{S}^{1 / 2}$, gauge invariance allows the following scalar coupling:

$$
V \supset \mathbf{6}_{S}^{1 / 2} \mathbf{6}_{S}^{1 / 2} \mathbf{6}_{S}^{-1 / 2} H^{-1 / 2},
$$

that explicitly breaks the accidental $\mathbb{Z}_{2}$ symmetry. The presence of this coupling enables the operator in figure 3- $(a)$, which induces fast decay processes of the neutral component of the multiplet, $\varphi^{0} \subset \mathbf{6}_{S}^{1 / 2}$, such as $\varphi^{0} \rightarrow W^{ \pm} W^{\mp} Z^{0}$. Since slow decays are - in general - not possible, its density is rapidly depleted and thus should be consistently included in the list of possible accidental matter representations. Note that this operator can be written even in the absence of additional representations, and so this conclusion proves to be true even in standard accidental matter scenarios.

Operators for $\mathbf{6}_{S}^{3 / 2}$ can also be written, but in contrast to the $\mathbf{6}_{S}^{1 / 2}$ case they require additional representations. The different decay operators are shown in figure 3, diagrams $(b)-(d)$. As can be seen, they all involve $\mathbb{Z}_{2}$-breaking couplings and therefore lead to fast decay processes of the lightest component of the multiplet, in this case $\varphi^{0} \subset \mathbf{6}_{S}^{3 / 2}$. Among those processes one can identify e.g. $\varphi^{0} \rightarrow W^{ \pm} W^{\mp} Z$ or $\varphi^{0} \rightarrow W^{ \pm} W^{\mp} h^{0}$. These couplings are of three kinds: independent of $\mathbf{6}_{S}^{3 / 2}$ and bilinear and linear in $\mathbf{6}_{S}^{3 / 2}$. Explicitly, for each operator, they are given by

$$
\begin{array}{lll}
\text { (b) : } & \boldsymbol{R}_{S}^{1 / 2} \boldsymbol{R}_{S}^{1 / 2} \boldsymbol{R}_{S}^{-1 / 2} H^{-1 / 2}, & \left(\boldsymbol{R}_{S}^{1 / 2}=\mathbf{4}_{S}^{1 / 2}, \mathbf{6}_{S}^{1 / 2}\right), \\
\text { (c) : } & \mathbf{6}_{S}^{3 / 2} \mathbf{6}_{S}^{3 / 2} \boldsymbol{R}_{S}^{-5 / 2} H^{-1 / 2}, & \left(\boldsymbol{R}_{S}^{-5 / 2}=\mathbf{4}_{S}^{-5 / 2}, \mathbf{6}_{S}^{-5 / 2}\right), \\
\text { (c) : } & \mathbf{6}_{S}^{3 / 2} \mathbf{5}_{S}^{-2} \mathbf{5}_{S}^{1} H^{-1 / 2}, & \\
\text { (d) : } & \mathbf{6}_{S}^{3 / 2} \boldsymbol{5}_{S}^{-1} \mathbf{5}_{S}^{-1} H^{1 / 2} . &
\end{array}
$$

As we will show in section 4.2, these couplings cover all possible UV completions associated with this representation, apart from one which involves the following representations: $\mathbf{5}_{S}^{2}$, $\mathbf{4}_{F}^{3 / 2}$ and $\mathbf{5}_{F}^{2}$ and for which we did not find a coupling enabling fast decay $\left(\mathbb{Z}_{2}\right.$-breaking coupling). This UV completion therefore has not been included in our analysis. Finally, for $\mathbf{6}_{S}^{5 / 2}$ several decay operators can be written too. Here, however, we present just a single one (see figure 3-(e)). The reason is that for this representation only few UV completions are consistent with our perturbativity criteria (see section 4.2), and this operator covers all of them. Being $\mathbb{Z}_{2}$-breaking it induces fast decays of the neutral component of the multiplet and so allows for a viable accidental $Y=5 / 2$ sextet.

\section{Radiative accidental matter}

We now turn to the discussion of UV completions of the operator in figure 1. For certain representations, in particular for higher-order ones, a certain UV completion can as well generate a $\operatorname{dim}=7$ or $\operatorname{dim}=9$ lepton-number-violating operator. In these cases one can find therefore regions in parameter space where the effective neutrino mass matrix 


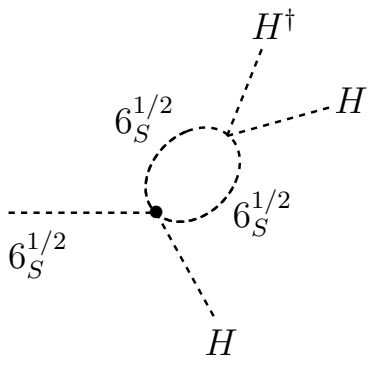

(a)

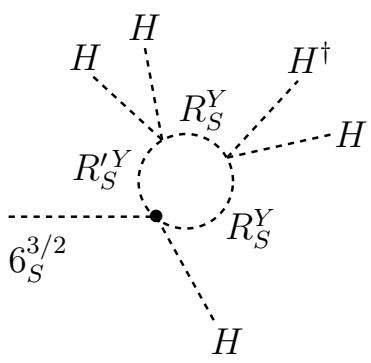

$(c)$

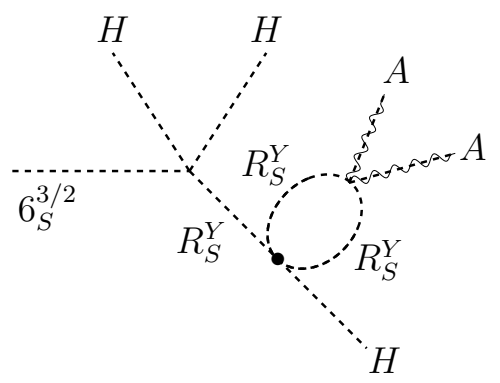

(b)

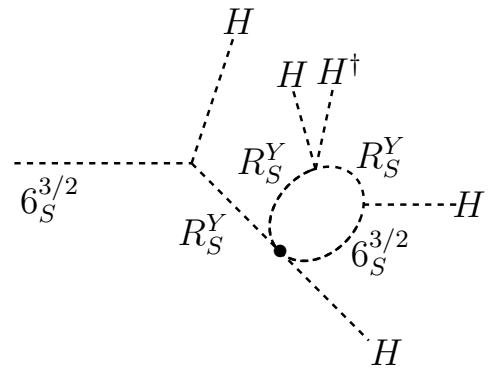

$(d)$

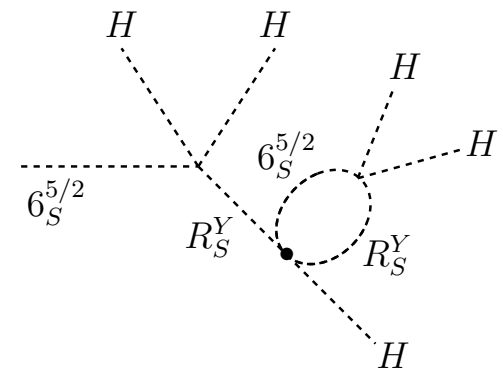

$(e)$

Figure 3. Loop-induced operators responsible for fast scalar sextet decays. Diagram $(a)$ is always present regardless of whether there are additional representations. For diagram $(b)$, $\boldsymbol{R}_{S}^{Y}=\mathbf{4}_{S}^{1 / 2}, \mathbf{6}_{S}^{1 / 2}$. The wiggly lines refer to $A=H$ for $\mathbf{4}_{S}^{1 / 2}$ and $A=V$, with $V$ a vector boson, for $\boldsymbol{6}_{S}^{1 / 2}$. In diagram $(c),\left\{\boldsymbol{R}_{S}^{Y}, \boldsymbol{R}_{S}^{\prime Y}\right\}=\left\{\boldsymbol{5}_{S}^{1}, \boldsymbol{5}_{S}^{2}\right\},\left\{\boldsymbol{4}_{S}^{5 / 2}, \boldsymbol{6}_{S}^{3 / 2}\right\},\left\{\boldsymbol{6}_{S}^{5 / 2}, \boldsymbol{6}_{S}^{3 / 2}\right\}$. In diagram $(d)$, $\boldsymbol{R}_{S}^{Y}=\mathbf{5}_{S}^{1}$. For diagram $(e), \boldsymbol{R}_{S}^{Y}=\mathbf{4}_{S}^{3 / 2}, \mathbf{6}_{S}^{3 / 2}$. The dots indicate $\mathbb{Z}_{2}$-breaking couplings, which ensure fast $\varphi^{0} \subset \mathbf{6}_{S}^{Y}$ decays thus making them suitable accidental matter representations.

receives contributions from several operators, or even where the neutrino mass matrix is entirely determined by the higher-order operator. Our assumption here is that $m_{\nu}$ is solely determined by the operator in figure 1, and this imposes a condition on the scale of the BSM degrees of freedom. The contribution to neutrino masses from the tree level dim $=7$ lepton-number breaking operator is $m_{\nu}^{\operatorname{dim}=7} \simeq v^{4} / \Lambda^{3}$. Thus, when compared with the oneloop contribution from the Weinberg operator it can be seen that $\Lambda \gtrsim 3 \mathrm{TeV}$ guarantees $m_{\nu}^{\operatorname{dim}=7}<m_{\nu}^{\operatorname{dim}=5}$, a condition satisfied by the BSM spectrum that defines the radiative accidental matter scenarios we are discussing (see figure 2). 


\subsection{Additional constraints: $\rho$ parameter and BBN}

Beyond the constraints we have already mentioned, there are other constraints one needs to bear in mind. Of particular relevance are those related with the breaking of the custodial symmetry, which place bounds on the mass of the accidental matter representations. A detailed analysis of these constraints has been presented in [17] and therefore here we discuss only those aspects that directly apply to radiative scenarios. In the limit $\sin \theta_{W} \rightarrow 0$ $\left(g^{\prime} \rightarrow 0\right)$, the weak gauge bosons $W^{ \pm}$and $Z$ transform as a triplet of an $\mathrm{SU}(2)_{L+R}$ global symmetry, which implies $m_{W^{ \pm}}=m_{Z}$. In that limit the SM $\rho$-parameter, defined as $\rho=m_{W}^{2} / m_{Z}^{2} \cos ^{2} \theta_{W}$, is one. Departures from this limit removes the gauge bosons mass degeneracy through $\cos \theta_{W}$, but still one finds $\rho_{\text {tree }}=1$, with small deviations induced by radiative corrections, that remain under control due to the $\mathrm{SU}(2)_{L+R}$ custodial symmetry. This value is consistent with its experimental value, $\rho_{\operatorname{Exp}}=1.0004_{-0.0004}^{+0.0003}[13]$.

Contributions to the $\rho$-parameter from BSM scalar fields that develop vevs can produce sizeable deviations from such value. For a set of scalars $\left\{\varphi_{T, Y}\right\}$ that acquire a vev, $\left\langle\varphi_{T, Y}\right\rangle$, and whose total weak isospins are $T$ and their hypercharges are $Y$, the tree level $\rho$-parameter reads $[46]$

$$
\rho_{\text {tree }}=\frac{\sum_{T, Y} c_{T, Y}\left[T(T+1)-Y^{2}\right]\left\langle\varphi_{j, Y}\right\rangle^{2}}{2 \sum_{Y} Y^{2}\left\langle\varphi_{j, Y}\right\rangle^{2}},
$$

where $c_{T, Y}=1\left(c_{T, Y}=1 / 2\right)$ for complex (real) fields and $Q=T_{3}+Y$. There is an infinite set of scalar fields that satisfy $\rho_{\text {tree }}=1$, determined by the condition

$$
\left(T+\frac{1}{2}\right)^{2}-3 Y^{2}=\frac{1}{4}
$$

The list of the viable scalar representations (that contain a neutral component) are determined by the following quantum numbers: $(T, Y)=$ $\{(0,0),(1 / 2, \pm 1 / 2),(3, \pm 2),(25 / 2, \pm 15 / 2), \cdots\} .{ }^{6} \quad$ Which shows that apart from the singlet, none of the other accidental matter scalar representation satisfies such condition and thus their vevs are subject to constraints. With only the Higgs and a single extra scalar field, expression (4.1) at order $\left\langle\varphi_{T, Y}\right\rangle^{2} / v^{2}$ can be cast according to

$$
\rho_{\text {tree }}-1 \simeq 2\left\{c_{T, Y}\left[T(T+1)-Y^{2}\right]-2 Y^{2}\right\} \frac{\left\langle\varphi_{T, Y}\right\rangle^{2}}{v^{2}},
$$

from which using the experimental upper limit for $\rho_{\operatorname{Exp}}$ one finds $\left\langle\varphi_{T, Y}\right\rangle / v \lesssim 1 \%$ [17]. This constraint is particularly important for accidental matter representations which develop an induced vev, namely the triplet and the quartet (the singlet does as well but its vev does not contribute to $\rho_{\text {tree }}$, as we have mentioned). For these representations this restriction translates into a lower bound on their masses, which can be (roughly) estimated from the minimization condition of the corresponding scalar potentials:

$$
V_{\mathbf{3}} \supset m_{3}^{2}\left|\mathbf{3}_{S}^{0}\right|^{2}+\mu \mathbf{3}_{S}^{0} H^{\dagger} H, \quad V_{\mathbf{4}} \supset m_{4}^{2}\left|\mathbf{4}_{S}^{Y}\right|^{2}+\lambda \mathbf{4}_{S}^{Y} S^{3},
$$

\footnotetext{
${ }^{6}$ The phenomenology of a $(T, Y)=(3, \pm 2)$ state that mixes with the SM Higgs doublet has been studied in ref. [47].
} 
with $S^{3}=H H^{\dagger} H^{\dagger}$ for $Y=1 / 2$ and $S^{3}=H^{\dagger} H^{\dagger} H^{\dagger}$ for $Y=3 / 2$. The result reads

$$
m_{3} \gtrsim 1568\left(\frac{\mu}{10^{2} \mathrm{GeV}}\right)^{1 / 2} \mathrm{GeV}, \quad m_{4} \gtrsim 246\left(\frac{\lambda}{10^{-2}}\right)^{1 / 2} \mathrm{GeV} .
$$

As can be seen, these values are consistent with radiative accidental matter models and in particular with the benchmark scenario we have chosen. It is worth emphasizing that in models where several of these states are found, these bounds will be more stringent with the values estimated to increase multiplicatively with the number of states.

Non-vanishing contributions to the $\rho$-parameter arise as well from radiative corrections to gauge boson masses. Mass splittings between the different components of a representation $\boldsymbol{R}$ lead to large radiative contributions, provided the splittings are large [48]. These splittings can arise from one-loop corrections (for fermions and scalars) and from off-diagonal terms in the tree level scalar mass matrices. The former are of order $\mathrm{MeV}$ [19] and so lead to negligible corrections to the $\rho$-parameter. The latter instead can involve large splittings and so can induce in turn sizeable deviations on $\rho$. However, when used to derive limits on scalar masses, the values found are less competitive than those in (4.5) or those coming from direct accelerator searches [17].

We now turn to the discussion of the constraints arising from BBN. Long-lived particles with lifetimes larger than $\sim 0.1$ seconds can significantly affect light-elements abundances through their electromagnetic and/or hadronic activity. Thus, consistency with observed light-elements abundances translates into constraints which lead e.g. to lower bounds on their masses/couplings $[49,50]$. Whether such constraints hold for the scenarios we are considering here depends on the lifetime of the different decay processes. For the representations in table 1 there are two types of decays. Intermultiplet decay processes in which heavier components of a multiplet undergo decays into lighter components, and decays of the lightest state (LS) into SM particles. The former are fast decay processes such as e.g. $R^{+} \rightarrow R^{0}+\pi^{+}$, and so they take place at early times well before BBN. The latter can be fast too, depending on whether the lightest particle can decay via renormalizable couplings or, in case it does not, on the cutoff scale. As it has been discussed in section 2.1, effective decay processes are driven by either $\operatorname{dim}=5$ or $\operatorname{dim}=6$ operators, for which the decay lifetimes for the LS can be estimated to be

$$
\begin{aligned}
& \tau_{\text {dim }=5} \simeq 9.8\left(\frac{\Lambda}{10^{6} \mathrm{GeV}}\right)^{2}\left(\frac{10^{3} \mathrm{GeV}}{m_{\mathrm{LS}}}\right)^{3} \mathrm{fs}, \\
& \tau_{\text {dim }=6} \simeq 18\left(\frac{\Lambda}{10^{6} \mathrm{GeV}}\right)^{4}\left(\frac{10^{3} \mathrm{GeV}}{m_{\mathrm{LS}}}\right)^{5} \mu \mathrm{s} .
\end{aligned}
$$

Note that this result assumes that the LS can directly decay via the non-renormalizable operator. However, this is not the case for $\mathbf{2}_{S}^{5 / 2}$ and $\mathbf{5}_{S}^{Y}(Y=1,2)$ which instead follow cascade decays mediated by off-shell heavier components of the representation. These processes have been studied in ref. [17] assuming $\Lambda=10^{15} \mathrm{GeV}$ and resulting in lifetimes amounting to $\sim 10^{3}$ seconds, but the rescaling of these results according to our cutoff scale lead to lifetimes comparable to those in (4.6). All in all, the decay processes of the accidental matter representations in radiative scenarios are fast, with the largest lifetimes amounting to at most microseconds, and so BBN constraints are of no relevance. 


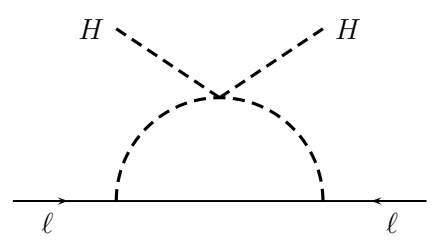

D1

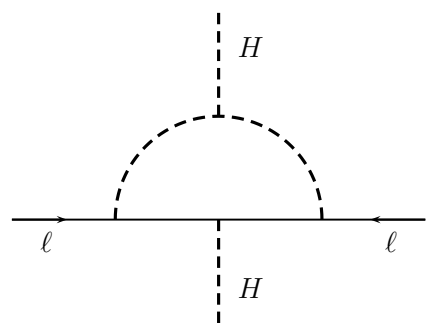

D3

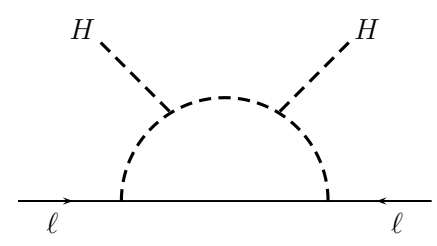

D2

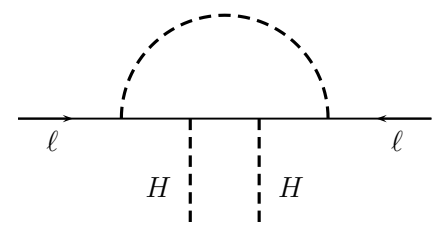

D4

Figure 4. One-loop diagrams for UV realizations of the dim=5 operator in figure 1.

\subsection{UV completions and perturbativity}

Our assumption is that at or above $10^{6} \mathrm{GeV}$ new degrees of freedom defining different UV completions for the operator in figure 1 become available. For a given representation the full set of UV completed models can be derived by considering the diagrams in figure 4, which correspond to all possible irreducible one-loop realizations of the operator in figure $1[51] .{ }^{7}$ We systematically fix the accidental matter representation within the loop in each of the diagrams and then fix the $\mathrm{SU}(2) \times \mathrm{U}(1)_{Y}$ quantum numbers of the remaining fields according to $\boldsymbol{R} \otimes \mathbf{2}=\boldsymbol{R} \pm 1$. UV completions involving hypercharge-zero fermion singlets or triplets or hypercharge-one scalar triplets are discarded, since they lead to seesaw neutrino masses ("seesaw filtering criterion"). These cases are found for $\mathbf{1}_{S}^{0}, \mathbf{3}_{S}^{0}$ and $\mathbf{4}_{F}^{Y}(Y=1 / 2,3 / 2)$. Rather than explicitly listing the resulting models, which amount to hundreds and that can be straightforwardly derived, we list in table 2 the representations which are needed in each case. These results are then used to identify those models that lead to the highest Landau pole scales.

For $\mathbf{1}_{S}^{0}$ all viable models are forbidden by the "seesaw filtering criterion". For $\mathbf{3}_{S}^{0}$ some models are found, but still the filtering criterion removes most of them leaving just few. For $\mathbf{4}_{F}^{Y}$, instead, this criterion removes only few models. Instead, in this case, a fairly large number of such models are found to be non-viable because they lead to non-perturbative effects below $10^{8} \mathrm{GeV}$, something found as well for other higher-order $\mathrm{SU}(2)$ representations.

Models that become non-perturbative below $10^{8} \mathrm{GeV}$, as defined by our benchmark scenario, are identified by using two-loop RGEs subject to the following energy thresholds (see appendix A):

- From $M_{Z}$ and up to $m_{\boldsymbol{R}}$ ( $\boldsymbol{R}$ being the accidental matter representation), that we take to be $1 \mathrm{TeV}$, the particle content is entirely given by the SM.

\footnotetext{
${ }^{7}$ Such classification does exist as well for the two-loop case, see ref. [52].
} 


\begin{tabular}{|c|c|c|c|c|c|c|c|c|c|c|c|c|}
\hline \multirow{3}{*}{$\begin{array}{c}\text { A.M. } \\
\mathbf{2}_{S}^{Y}\end{array}$} & \multirow{2}{*}{\multicolumn{3}{|c|}{ Fermion sector }} & \multirow{2}{*}{\multicolumn{5}{|c|}{ Scalar sector }} & \multicolumn{4}{|c|}{ \# of models } \\
\hline & & & & & & & & & \multirow{2}{*}{$\frac{\text { D1 }}{12}$} & \multirow{2}{*}{$\begin{array}{c}\text { D2 } \\
24\end{array}$} & \multirow{2}{*}{\begin{tabular}{|c|} 
D3 \\
17
\end{tabular}} & \multirow{2}{*}{\begin{tabular}{|c|} 
D4 \\
10
\end{tabular}} \\
\hline & $\mathbf{1}_{F}^{Y \pm 1 / 2}$ & $\mathbf{2}_{F}^{Y}$ & $\mathbf{3}_{F}^{Y \pm 1 / 2} \quad \mathbf{4}_{F}^{Y}$ & $\mathbf{1}_{S}^{Y \pm 1 / 2}$ & $\mathbf{2}_{S}^{Y \pm 1}$ & $\mathbf{3}_{S}^{Y \pm 1 / 2}$ & $\mathbf{4}_{S}^{Y \pm 1}$ & & & & & \\
\hline $\mathbf{3}_{S}^{0}$ & $\mathbf{2}_{F}^{1 / 2}$ & $4_{F}^{1 / 2}$ & $\mathbf{5}_{F}^{0}$ & $\mathbf{1}_{S}^{1}$ & $\mathbf{2}_{S}^{1 / 2}$ & $4_{S}^{1 / 2}$ & $\mathbf{5}_{S}^{1}$ & & 2 & 3 & 1 & 1 \\
\hline $\mathbf{4}_{S}^{Y}$ & $\mathbf{2}_{F}^{Y}$ & $\mathbf{3}_{F}^{Y \pm 1 / 2}$ & $\mathbf{4}_{F}^{Y} \quad \mathbf{5}_{F}^{Y \pm 1 / 2} \mathbf{6}_{F}^{Y}$ & $\mathbf{2}_{S}^{Y \pm 1}$ & $\mathbf{3}_{S}^{Y \pm 1 / 2}$ & $\mathbf{4}_{S}^{Y \pm 1}$ & $\mathbf{5}_{S}^{Y \pm 1 / 2}$ & $\mathbf{6}_{S}^{Y \pm 1}$ & 14 & 21 & 15 & 8 \\
\hline $\mathbf{5}_{S}^{Y}$ & $\mathbf{3}_{F}^{Y}$ & $\mathbf{4}_{F}^{Y \pm 1 / 2}$ & $\mathbf{6}_{F}^{Y \pm 1 / 2} \mathbf{7}_{F}^{Y}$ & $\mathbf{3}_{S}^{Y \pm 1}$ & $\mathbf{4}_{S}^{Y \pm 1 / 2}$ & $\mathbf{5}_{S}^{Y \pm 1}$ & $\mathbf{6}_{S}^{Y \pm 1 / 2}$ & $\mathbf{7}_{S}^{Y \pm 1}$ & 11 & 21 & 13 & 8 \\
\hline $\mathbf{6}_{S}^{Y}$ & $\mathbf{4}_{F}^{Y}$ & $\boldsymbol{5}_{F}^{Y \pm 1 / 2}$ & $\mathbf{6}_{F}^{Y} \quad \mathbf{7}_{F}^{Y-1 / 2}$ & $4_{S}^{Y}$ & $\mathbf{4}_{S}^{Y \pm 1}$ & $\boldsymbol{5}_{S}^{Y \pm 1 / 2}$ & $\mathbf{6}_{S}^{Y \pm 1}$ & $\mathbf{7}_{S}^{Y-1 / 2}$ & 9 & 15 & 5 & 1 \\
\hline $\mathbf{4}_{F}^{Y}$ & $\mathbf{3}_{F}^{Y \pm 1 / 2}$ & $\mathbf{4}_{F}^{Y \pm 1}$ & $\mathbf{5}_{F}^{Y \pm 1 / 2} \mathbf{6}_{F}^{Y-1}$ & $\mathbf{2}_{S}^{Y}$ & $\mathbf{3}_{S}^{Y \pm 1 / 2}$ & $4_{S}^{Y}$ & $\mathbf{5}_{S}^{Y \pm 1 / 2}$ & $\mathbf{6}_{S}^{Y}$ & 4 & 5 & 13 & 19 \\
\hline $\mathbf{5}_{F}^{0}$ & $\mathbf{3}_{F}^{1}$ & $4_{F}^{1 / 2}$ & $\mathbf{6}_{F}^{1 / 2}$ & $\mathbf{3}_{S}^{0}$ & $4_{S}^{1 / 2}$ & $\mathbf{5}_{S}^{0}$ & $\mathbf{6}_{S}^{1 / 2}$ & $\mathbf{7}_{S}^{0}$ & 3 & 5 & 5 & 7 \\
\hline
\end{tabular}

Table 2. Representations needed for the construction of UV completions of the operator in figure 1 for the different accidental matter representations in table 1 . The last four columns refer to the number of viable models determined by the UV completions in figure 4 and selected according to the condition that the particle content of the model does not enable type-I, type-II or type-III seesaw and that $\alpha_{1,2}$ remain perturbative at least up to $10^{8} \mathrm{GeV}$.

- From $m_{\boldsymbol{R}}$ and up to $10^{6} \mathrm{GeV}$, where according to our benchmark scenario the UV completions for the operator in figure 1 kick in, the particle content is determined by the $\mathrm{SM}+\boldsymbol{R}$.

- Above $10^{6} \mathrm{GeV}$, RGE running takes into account the $\mathrm{SM}+\boldsymbol{R}+\boldsymbol{R}^{\prime}$, with $\boldsymbol{R}^{\prime}$ referring to representations that define the UV completions.

For low-order $\mathrm{SU}(2)$ representations up to the triplet, Landau poles are found at rather high scales, ranging from $10^{11} \mathrm{GeV}$ up to $10^{19} \mathrm{GeV}$, with the exception being few models for $\mathbf{2}_{S}^{5 / 2}$ for which $\alpha_{1}=g_{1}^{2} / 4 \pi$ develops a Landau pole at $\sim 10^{8} \mathrm{GeV}$, due to the large hypercharges of the extra representations. Thus, apart from this representation, all low-order radiative accidental matter models are consistent with perturbativity up to $10^{8} \mathrm{GeV}$.

For higher-order accidental matter representations this behavior remains like that for the $4_{S}^{1 / 2}$ and also for $4_{S}^{3 / 2}$, but for $4_{S}^{3 / 2}$ some models fail to pass the perturbativity condition. For $\mathbf{5}_{S}^{0}$ all models are consistent with perturbativity and $\Lambda_{\mathrm{LP}}^{\text {highest }}$ remains at $10^{13} \mathrm{GeV}$, depending on the model category (defined by diagrams D1-D2). For $\mathbf{5}_{S}^{Y}(Y=1,2)$, the largest Landau pole scales are somehow degraded with values even as low as $10^{9} \mathrm{GeV}$ for $\mathbf{5}_{S}^{2}$, again depending on the model category. In these cases many models reach Landau poles well below $10^{8} \mathrm{GeV}$, and so fail to pass the pertubativity cut. This trend persists for the remaining accidental matter representations, including the $\mathbf{4}_{F}^{Y}$, being very pronounced for $\mathbf{6}_{S}^{3 / 2}$ and $\mathbf{6}_{S}^{5 / 2}$ for which there are model categories that do not contain any model satisfying the perturbativity criterion. These results are summarized in figure 5, where we have plotted the range for the Landau pole scale for the different accidental matter representations in each category model.

Among all the viable models we select those for which the Landau pole scale is the largest. These radiative accidental matter models are arguably the most compelling ones. For models involving $\mathbf{3}_{S}^{0}$ and $\mathbf{2}_{S}^{3 / 2}$ several setups for which $\Lambda_{\mathrm{LP}} \gtrsim 10^{19} \mathrm{GeV}$ are found. 


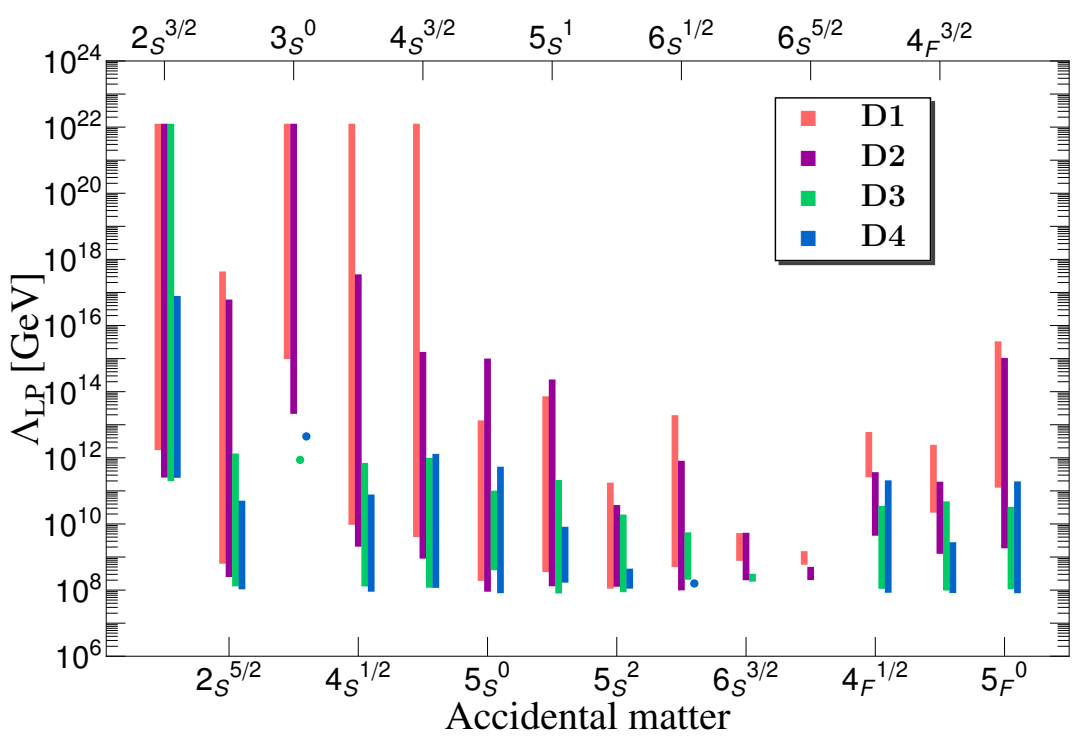

Figure 5. Range of the Landau pole scales for the UV models of the operator in figure 1, displayed according to the category models defined by diagrams D1-D4 in figure 4. Bars exceeding $10^{19} \mathrm{GeV}$ indicate that the Landau pole is reached above $M_{\text {Planck }}$, and do not refer to any precise value. For some representations/models only a point is displayed: for $\mathbf{3}_{S}^{0}$ a single model (D3 and D4) is possible after the "seesaw filtering criterion" is applied, for $\mathbf{6}_{S}^{1 / 2}(\mathbf{D} 4)$ and $\mathbf{6}_{S}^{3 / 2}$ (D3) only a single model reaches a Landau pole above $10^{8} \mathrm{GeV}$. Furthermore, for $\mathbf{6}_{S}^{3 / 2}$ all models in category $\mathbf{D} 4$ reach a Landau pole below $10^{8} \mathrm{GeV}$, while for $\mathbf{6}_{S}^{5 / 2}$ all models in $\mathbf{D} 3$ and $\mathbf{D} 4$ do so.

In these cases the selection criterion is that of the model involving the least number of representations. In almost all cases the corresponding models are D1-based, something somehow expected since these UV completions involve the least number of fermions and so gauge couplings run slower. In all cases as well we have found that $\alpha_{2}$ reaches the Landau pole before $\alpha_{1}$ does, with a single exception given by $\mathbf{2}_{S}^{5 / 2}$. The models are shown in table 3 , where it can be seen that models with $\mathbf{5}_{S}^{2}, \mathbf{6}_{S}^{3 / 2}$ and $\mathbf{6}_{S}^{5 / 2}$ are somehow disfavored by the relatively low Landau pole scale.

\subsection{Lepton flavor violation: generic approach}

In this section we quantify the expected size of SM charged-lepton flavor violating (CLFV) radiative processes in the models depicted in table $3 .^{8}$ Three body CLFV decay processes, in particular $\mu^{+} \rightarrow e^{+} e^{-} e^{+}$, and $\mu-e$ conversion in nuclei are relevant as well due to the large sensitivity of near-future experimental facilities: $M u 3 e$ at PSI aims at measuring $\mu^{+} \rightarrow e^{+} e^{-} e^{+}$to a precision of $10^{-16}$ [29, 30], while PRISM/PRIME at J-PARC $\mu-e$ conversion in nuclei down to $10^{-18}[31,32]$. Results for these processes will be presented elsewhere [55].

Rather than sticking to a particular realization or analyzing them all we make use of the fact that the problem follows a generic treatment. Since most of the compelling models

\footnotetext{
${ }^{8} \mathrm{CLFV}$ in models with higher-order $\mathrm{SU}(2)$ representations, which are among the models shown in table 3 , have been considered in e.g. refs. [45, 53, 54].
} 


\begin{tabular}{|c|cc|c|c|}
\hline Acc. Matter & \multicolumn{2}{|c|}{ Extra representations } & Model & $\Lambda_{\mathrm{LP}}$ \\
\hline $\mathbf{2}_{S}^{3 / 2}$ & $\mathbf{1}_{F}^{1}$ & $\mathbf{2}_{S}^{1 / 2}$ & $\mathbf{D} \mathbf{1}$ & $>10^{19} \mathrm{GeV}$ \\
\hline $\mathbf{2}_{S}^{5 / 2}$ & $\mathbf{1}_{F}^{2}$ & $\mathbf{2}_{S}^{3 / 2}$ & $\mathbf{D} \mathbf{1}$ & $3.41 \times 10^{17} \mathrm{GeV}$ \\
\hline $\mathbf{3}_{S}^{0}$ & $\mathbf{2}_{F}^{1 / 2}$ & $\mathbf{1}_{S}^{1}$ & $\mathbf{D} \mathbf{1}$ & $>10^{19} \mathrm{GeV}$ \\
\hline $\mathbf{4}_{S}^{1 / 2}$ & $\mathbf{3}_{F}^{1}$ & $\mathbf{2}_{S}^{3 / 2}$ & $\mathbf{D} \mathbf{1}$ & $>10^{19} \mathrm{GeV}$ \\
\hline $\mathbf{4}_{S}^{3 / 2}$ & $\mathbf{3}_{F}^{1}$ & $\mathbf{2}_{S}^{1 / 2}$ & $\mathbf{D 1}$ & $>10^{19} \mathrm{GeV}$ \\
\hline $\mathbf{5}_{S}^{0}$ & $\mathbf{5}_{F}^{0}$ & $\mathbf{4}_{S}^{1 / 2}$ & $\mathbf{D} \mathbf{2}$ & $7.94 \times 10^{14} \mathrm{GeV}$ \\
\hline $\mathbf{5}_{S}^{1}$ & $\mathbf{4}_{F}^{1 / 2}$ & $\mathbf{3}_{S}^{0}$ & $\mathbf{D} \mathbf{2}$ & $5.74 \times 10^{13} \mathrm{GeV}$ \\
\hline $\mathbf{5}_{S}^{2}$ & $\mathbf{6}_{F}^{3 / 2}$ & $\mathbf{5}_{S}^{1}$ & $\mathbf{D 1}$ & $1.03 \times 10^{9} \mathrm{GeV}$ \\
\hline $\mathbf{6}_{S}^{1 / 2}$ & & $\mathbf{5}_{F}^{0}$ & $\mathbf{D} \mathbf{1}$ & $1.54 \times 10^{13} \mathrm{GeV}$ \\
\hline $\mathbf{6}_{S}^{3 / 2}$ & $\mathbf{5}_{F}^{1}$ & $\mathbf{4}_{S}^{1 / 2}$ & $\mathbf{D} \mathbf{1}$ & $4.19 \times 10^{9} \mathrm{GeV}$ \\
\hline $\mathbf{6}_{S}^{5 / 2}$ & $\mathbf{5}_{F}^{2}$ & $\mathbf{6}_{S}^{3 / 2}$ & $\mathbf{D} \mathbf{1}$ & $7.4 \times 10^{8} \mathrm{GeV}$ \\
\hline $\mathbf{4}_{F}^{1 / 2}$ & $\mathbf{3}_{S}^{0}$ & $\mathbf{5}_{S}^{1}$ & $\mathbf{D} \mathbf{1}$ & $4.76 \times 10^{12} \mathrm{GeV}$ \\
\hline $\mathbf{4}_{F}^{3 / 2}$ & $\mathbf{3}_{S}^{2}$ & $\mathbf{5}_{S}^{1}$ & $\mathbf{D} \mathbf{1}$ & $1.95 \times 10^{12} \mathrm{GeV}$ \\
\hline $\mathbf{5}_{F}^{0}$ & & $\mathbf{4}_{S}^{1 / 2}$ & $\mathbf{D} \mathbf{1}$ & $2.63 \times 10^{15} \mathrm{GeV}$ \\
\hline
\end{tabular}

Table 3. Radiative accidental matter models for which the Landau pole is reached at the highest possible scale. Apart from $\mathbf{5}_{S}^{0}$ and $\mathbf{5}_{S}^{1}$, all models are D1-based. This list therefore defines the most compelling radiative accidental matter models. Note that the relatively low Landau pole scale for $\mathbf{5}_{S}^{2}, \mathbf{6}_{S}^{3 / 2}$ and $\mathbf{6}_{S}^{5 / 2}$ disfavors these models.

are determined by $\mathbf{D} \mathbf{1}$ diagrams, we will focus on such models for which a schematic Lagrangian can be used for the discussion:

$$
\mathcal{L}=\overline{\ell c}_{i} Y_{i \alpha} P_{L} F_{\alpha} S_{a}+\lambda_{a b} S_{a} S_{b} H H+\bar{F}_{\alpha} Y_{j \alpha} P_{L} \ell_{j} S_{b}+\text { H.c. },
$$

where $F(S)$ refer to vectorlike fermions (scalars) in any of the representations displayed in table 3, and so $\mathrm{SU}(2)$ contractions are assumed. Sum over lepton flavor and fermion generation indices is understood, while $a$ and $b$ just label different scalars. Note that in addition to the terms in (4.8), there are also pure scalar and fermion mass terms (that can be of Majorana type if $Y(F)=0$ ) which we are not writing, but are essential since they determine scalar mixing and eventually fermion mixing too. Furthermore, we are assuming for simplicity that Yukawa couplings are the same regardless of the scalar to which the fermion bilinear is coupled.

We take two generations of vectorlike fermions, which is the minimal number required to generate two non-zero light neutrino masses in this simplified setup we have assumed. This is a direct consequence of assuming same Yukawa couplings, for different Yukawa couplings a single fermion will suffice. Note that in models where more than a single 
fermion is needed the Landau pole scale will be below the numbers quoted in table 3 . Since in this section we aim just at showing that CLFV effects are within reach and we are not specifying quantum numbers we stick to the values in table 3 . Components of the scalar multiplets (that we assume there is only one copy per representation) with the same electric charge $Q$ mix through the scalar coupling in (4.8). Depending on $Q$, their mass eigenstates will contribute to the neutrino mass operator. One can distinguish for example: $(i)$ one pair of scalar mass eigenstates with electric charge $Q$ determine the neutrino mass matrix, (ii) two pairs with electric charges $Q$ and $Q^{\prime}$ are responsible for the operator. Just to mention a couple of cases, in the model for $\mathbf{2}_{S}^{3 / 2}$, states $\varphi^{+} \subset \mathbf{2}_{S}^{3 / 2}$ and $\rho^{+} \subset \mathbf{2}_{S}^{1 / 2}$ mix, and their mass eigenstates $\left(S_{1}^{+}, S_{2}^{+}\right)$lead to two diagrams that determine the neutrino mass matrix. In contrast, in case $\mathbf{4}_{S}^{1 / 2}$, states $\varphi^{+} \subset \mathbf{2}_{S}^{3 / 2}$ and $\rho^{+} \subset \mathbf{4}_{S}^{1 / 2}$ and $\varphi^{++} \subset \mathbf{2}_{S}^{3 / 2}$ and $\rho^{++} \subset \mathbf{4}_{S}^{1 / 2}$ mix and define the eigenstates $\left(S_{1}^{+}, S_{2}^{+}\right)$and $\left(S_{1}^{++}, S_{2}^{++}\right)$that in turn lead to the neutrino mass matrix.

Therefore, after rotation to the scalar mass eigenstate bases and regardless of the radiative accidental matter model, the neutrino mass matrix consist of a series of diagrams that guarantee cancellation of the divergent piece of the Passarino-Veltman (PV) function $B_{0}\left(0, m_{S_{a}}^{2}, m_{F}\right)$ [56]. The finite piece defines in turn the neutrino mass matrix that reads:

$$
\left(m_{\nu}\right)_{i j}=\frac{1}{16 \pi^{2}} \sum_{\alpha=1,2} m_{F_{\alpha}} Y_{i \alpha} Y_{j \alpha} \sum_{a} \Theta_{a}^{2} B_{0}^{\mathrm{fin}}\left(0, m_{S_{a}}^{2}, m_{F_{\alpha}}^{2}\right)
$$

where $\Theta_{a}^{2}$ parameterizes scalar mixing and satisfies $\sum_{a} \Theta_{a}^{2}=0$. In the simplest case $a=1,2, \Theta_{a}^{2}=(-)^{a} \sin \theta \cos \theta(\theta$ the mixing angle) and so the finite piece of the PV function under the sum over $a$ can be written according to

$$
B_{0}^{\mathrm{fin}}\left(0, m_{S_{a}}^{2}, m_{F_{\alpha}}^{2}\right) \rightarrow B_{0}^{\mathrm{fin}}\left(0, m_{S_{a}}^{2}, m_{F_{\alpha}}^{2}\right)=\frac{m_{S_{a}}^{2}}{m_{S_{a}}^{2}-m_{F_{\alpha}}^{2}} \log \left(\frac{m_{S_{a}}^{2}}{m_{F_{\alpha}}^{2}}\right) .
$$

Note that the neutrino mass matrix is rank-two and so there is a massless light neutrino, as already anticipated. To determine the Yukawa structure required by neutrino oscillation data [42-44], that combined with the mass scale for the fermions and scalars determines the LFV rates, we start by recasting the neutrino mass matrix according to

$$
\boldsymbol{m}_{\boldsymbol{\nu}}=\boldsymbol{Y} \cdot \widehat{\boldsymbol{F}} \cdot \boldsymbol{Y}^{T}
$$

where $\boldsymbol{Y}$ is a $3 \times 2$ Yukawa coupling matrix whose entries are given by $Y_{i \alpha}$ and $\widehat{\boldsymbol{F}}$ is a dimensionful $2 \times 2$ diagonal matrix whose non-vanishing entries read:

$$
\widehat{\boldsymbol{F}}_{\alpha}=-\frac{\Theta^{2} m_{F_{\alpha}}}{16 \pi^{2}}\left[\frac{m_{S_{1}}^{2}}{m_{S_{1}}^{2}-m_{F_{\alpha}}^{2}} \log \left(\frac{m_{S_{1}}^{2}}{m_{F_{\alpha}}^{2}}\right)-\frac{m_{S_{2}}^{2}}{m_{S_{2}}^{2}-m_{F_{\alpha}}^{2}} \log \left(\frac{m_{S_{2}}^{2}}{m_{F_{\alpha}}^{2}}\right)\right] .
$$

With the mass matrix written as in (4.11) the Yukawas can be then parameterized à la Casas-Ibarra [57], namely

$$
\boldsymbol{Y}=\boldsymbol{U}^{*} \widehat{\boldsymbol{m}}_{\boldsymbol{\nu}}{ }^{1 / 2} \boldsymbol{R}^{T} \widehat{\boldsymbol{F}}^{-1 / 2}
$$


Here $\boldsymbol{U}$ is the lepton mixing matrix and $\boldsymbol{R}$ is a $3 \times 2$ orthogonal complex matrix that can be written as [58]

$$
\boldsymbol{R}=\left(\begin{array}{ccc}
0 & \cos z & \sin z \\
0 & -\sin z & \cos z
\end{array}\right)
$$

where $z$ is a complex angle. With the aid of eq. (4.13), radiative CLFV processes can be calculated by fixing the scalar mass spectrum and using neutrino data.

The branching fractions for these processes can be written according to [59]

$$
\operatorname{Br}\left(l_{i} \rightarrow l_{j} \gamma\right)=\frac{\Gamma\left(l_{i} \rightarrow l_{j} \gamma\right)}{\Gamma_{\text {Tot }}^{l_{i}}}=\frac{m_{i}^{5}}{4096 \pi^{5} \Gamma_{\text {Tot }}^{l_{i}}}\left|\sum_{a, \alpha=1}^{2} \frac{Y_{i \alpha} Y_{j \alpha}^{*}}{m_{S_{a}}^{2}}\left[Q_{F} G_{1}\left(t_{\alpha a}\right)+\left(Q_{F}+1\right) G_{2}\left(t_{\alpha a}\right)\right]\right|^{2}
$$

with $t_{\alpha a}=m_{F_{\alpha}}^{2} / m_{S_{a}}^{2}, \Gamma_{\text {Tot }}^{l_{i}}$ the total decay width of $l_{i}$ and the loop functions given by

$$
G_{1}(t)=\frac{2+3 t-6 t^{2}+t^{3}+6 \log t}{12(t-1)^{4}} \quad \text { and } \quad G_{2}(t)=\frac{1-6 t-3 t^{2}+2 t^{3}-6 t^{2} \log t}{12(t-1)^{4}}
$$

With these results at hand we calculated the muon and tau decay branching ratios as a function of the heaviest fermion mass. For that aim we fixed fermion masses according to $m_{F_{2}}-m_{F_{1}}=500 \mathrm{GeV}$, while randomly varying $m_{F_{2}}$ in the range $\left[10^{4}, 10^{9}\right] \mathrm{GeV}$. The scalar spectrum according to $m_{S_{2}}=10^{3} \mathrm{GeV}$ and $m_{S_{1}}=500 \mathrm{GeV}$, neutrino low-energy observables to their best-fit point values [43] and scalar mixing in the range $\Theta^{2}=\left[10^{-12}, 10^{-8}\right]$, randomly varied too. For simplicity we have taken $z$ real and equal to $\pi / 10$. We have checked that the result is pretty insensitive to the value of $z$ as long as $z$ is real. The result as well is not very sensitive to the value of $Q_{F}$ provided $Q_{F}<2$. For all points in the scan we have checked $\max (\boldsymbol{Y})<\sqrt{4 \pi}$. Note that this parameter choice has nothing special and has been taken just to exemplify the typical CLFV behavior one expects in these scenarios.

Figure 6 shows the result for $\mu \rightarrow e \gamma$ as a function of the heavy fermion mass. It shows that for a reasonable mass range this process falls within the region determined by MEG near-future sensitivities [60]. The result is representative of what is expected for the radiative accidental matter models listed in table 3 . Since it has been done without sticking to a particular realization, it is of course subject to numerical variations determined by the details of the model and its particular behavior with relevant parameters. As can be seen from eqs. (4.15) and (4.16), the LFV observable depends on $1 / m_{F}^{4}$. The apparently different behavior of the lower limit in figure 6 is a result of the constraints imposed on the parameter scan (used to improve the plot layout), in particular those on $\Theta$, and thus has nothing to do with any physical observable effect. For radiative tau decay processes we have found that $\tau \rightarrow e \gamma$ lies one order of magnitude below SuperKEKB sensitivities [61], while $\tau \rightarrow \mu \gamma$ can be within the range of observability if the mass gap between $\boldsymbol{R}$ (the accidental matter representation) and the radiative $\operatorname{dim}=5$ operator UV completion is not large, $\mathcal{O}\left(m_{\mathrm{UV}}\right) \sim 10 m_{\boldsymbol{R}}$. This, however, would lead to a lower $\Lambda_{\mathrm{LP}}$ scale and so according to our approach is disfavored. 


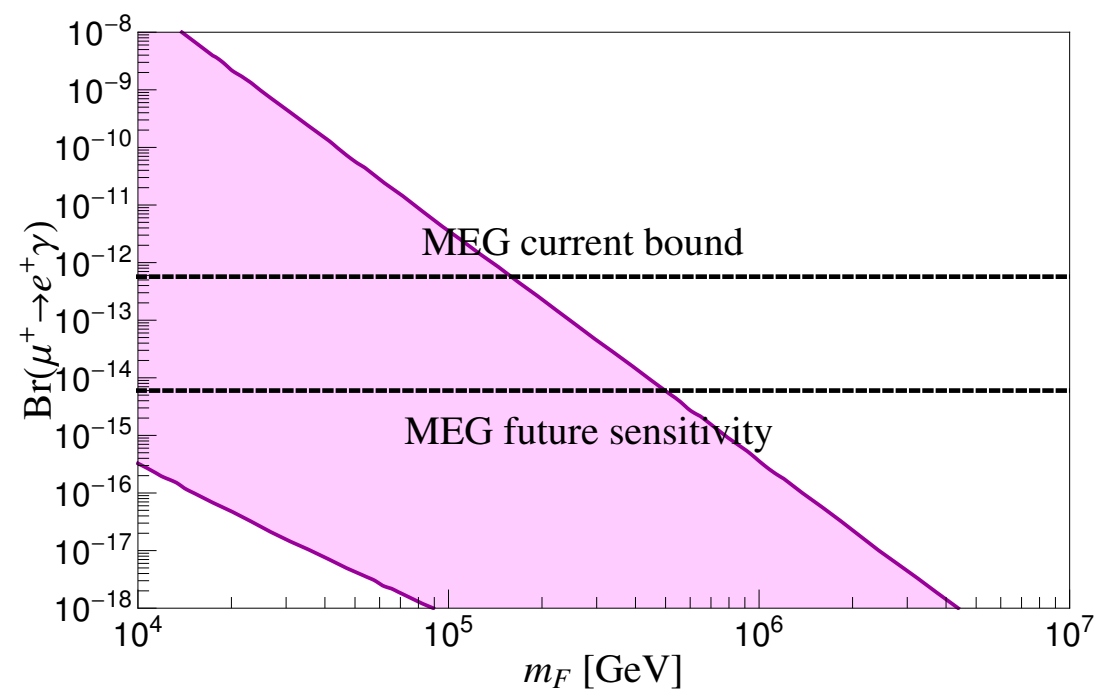

Figure 6. Decay branching fraction for $\mu \rightarrow e \gamma$ as a function of a generic fermion mass $m_{F}$. The upper horizontal line indicates current MEG bound [12], whereas the lower line MEG future sensitivity [60]. This result has been derived by assuming that the process is mediated by a pair of charged fermions (scalars) with $Q_{F}=+1\left(Q_{S}=+2\right)$. The result is representative of what is expected from the radiative accidental matter models listed in table 3 (compelling radiative accidental matter models), with possible numerical variations determined by the specific model and/or parameter space region.

\section{Conclusions}

Accidental matter models involve BSM degrees of freedom that automatically preserve the SM accidental and approximate symmetries up to a certain cutoff scale. Assuming that this scale is determined by lepton number violation and in the absence of a "nonconventional" suppression mechanism, this scale is fixed at $\sim 10^{15} \mathrm{GeV}$. In this paper, we studied accidental matter models assuming that the accidental matter representations play an active role in neutrino mass generation (radiative accidental matter models), something that we have shown necessary leads to radiatively induced neutrino masses and therefore to a suppressed LNV scale that can naturally be as low as $10^{6} \mathrm{GeV}$.

By defining a benchmark radiative accidental matter scenario (see figure 2), we have shown that in this new context higher-order accidental matter representations are no longer cosmologically stable. In particular, we have shown that this observation combined with a lower perturbative scale enables $Y=1 / 2,3 / 2,5 / 2$ scalar sextets. By considering EW and BBN constraints, we have as well derived lower bounds on the masses of various accidental matter representations, showing that masses of about $1 \mathrm{TeV}$ always imply a consistent picture.

We have identified the different UV completions of the radiative dimension five operator in figure 1. We have systematically studied their perturbative behavior and filtered viable models according to whether $\alpha_{1}$ and $\alpha_{2}$ remain perturbative up to at least $10^{8} \mathrm{GeV}$, our results are summarized in figure 5. From this classification we have determined the most 
compelling radiative accidental matter models by the condition that the Landau pole scale is the largest possible. Our results are listed in table 3. For the resulting models we have studied in a fairly model-independent way the typical size of radiative CLFV effects. According to our findings, $\mu \rightarrow e \gamma$ is within reach for all models provided the UV completion of the operator in figure 1 does not exceed $\sim 10^{6} \mathrm{GeV}$. In contrast, for $\tau \rightarrow \mu \gamma$ observability requires a somewhat BSM compressed spectrum that in turn leads to a lower Landau pole scale, something disfavored by our approach but not excluded. Thus, these setups offer a rich LFV phenomenology that increases their experimental testability and which motivates further phenomenological studies of the resulting setups [55].

\section{Acknowledgments}

We would like to thank Luca di Luzio for useful comments. This work was supported by the "Fonds de la Recherche Scientifique-FNRS" under grant number 4.4501.15. The work of C.S. is supported by the "Université de Liège" and the EU in the context of the MSCA-COFUND-BeIPD project.

\section{A Two-loop renormalization group equations}

The evolution of the gauge coupling constants, $\alpha_{i}(i=1,2,3)$, with the energy scale $\mu$, at two-loop level, is given by the RGEs

$$
\mu \frac{d}{d \mu} \alpha_{i}^{-1}=-\frac{b_{i}}{2 \pi}-\frac{1}{8 \pi^{2}} \sum_{j} b_{i j} \alpha_{j},
$$

with $\alpha_{1}=5 / 3 \alpha_{y}, \alpha_{i}=g_{i}^{2} / 4 \pi$ and $b_{i}$ and $b_{i j}$ the one- and two-loop beta functions, respectively. For a generic multiplet with $G_{\mathrm{SM}}=\mathrm{SU}(3)_{C} \times \mathrm{SU}(2)_{L} \times \mathrm{U}(1)_{Y}$ quantum numbers $\left(d_{3}, d_{2}, y\right), b_{i}$ and $b_{i j}$ are given by $[62,63]$

$$
b_{i}= \begin{cases}-\frac{11}{3} C_{i}, & \text { for gauge bosons } \\ \frac{4}{3} f T_{i} d_{k} d_{m}, & \text { for fermions } \\ \frac{1}{3} s T_{i} d_{k} d_{m}, & \text { for scalars }\end{cases}
$$

and

$$
b_{i j}= \begin{cases}-\frac{34}{3} C_{i}^{2} \delta_{i j}, & \text { for gauge bosons } \\ f\left[4 C_{i}+\frac{20}{3} C_{i}(\mathrm{Adj})\right] T_{i} d_{k} d_{m} \delta_{i j}+\left[4 f C_{j} T_{i} d_{k} d_{m}\right]_{i \neq j}, & \text { for fermions } \\ s\left[4 C_{i}+\frac{2}{3} C_{i}(\mathrm{Adj})\right] T_{i} d_{k} d_{m} \delta_{i j}+\left[4 s C_{j} T_{i} d_{k} d_{m}\right]_{i \neq j}, & \text { for scalars }\end{cases}
$$

with $s=1 / 2(s=1)$ for real (complex) scalars, while $f=1 / 2(f=1)$ for Weyl (Dirac) fermions. $d_{k}$ and $d_{m}$ are the dimensions of the multiplet with respect to the remaining subgroups, $G_{k}$ and $G_{m}$, and $m, k \neq i$. The quantities $C_{i}$ and $T_{i}$ are respectively the Casimir invariant and the Dynkin index for the multiplet under consideration with respect to the subgroup $G_{i} \subset G_{\mathrm{SM}}$. The Casimir for the adjoint representation of $G_{i}$ is denoted by $C_{i}(\mathrm{Adj})$. 


\begin{tabular}{|c|cccccccc|}
\hline & $\mathbf{1}$ & $\mathbf{2}$ & $\mathbf{3}$ & $\mathbf{4}$ & $\mathbf{5}$ & $\mathbf{6}$ & $\mathbf{7}$ & $\mathbf{8}$ \\
\hline$T_{2}$ & 0 & $1 / 2$ & 2 & 5 & 10 & $35 / 2$ & 28 & 42 \\
$C_{2}$ & 0 & $3 / 4$ & 2 & $15 / 4$ & 6 & $35 / 4$ & 12 & $63 / 4$ \\
\hline
\end{tabular}

Table 4. Dynkin index $\left(T_{2}\right)$ and Casimir invariant $\left(C_{2}\right)$ for $\mathrm{SU}(2)$ representations up to dimension eight.

The Casimir invariants and Dynkin indices $\left(C_{2}\right.$ and $\left.T_{2}\right)$ for $\mathrm{SU}(2)$ representations up to octets are given in table 4 . For $\mathrm{U}(1)_{Y}$ one has instead $T_{1}=y^{2}$. Note that since all the multiplets we consider are color singlets they do not contribute to $\mathrm{SU}(3)$ running and hence we do not specify neither $C_{3}$ nor $T_{3}$. For the gauge couplings at $M_{Z}=91.188 \mathrm{GeV}$ we use [13]:

$$
\alpha_{1}\left(M_{Z}\right)=0.016923, \quad \alpha_{2}\left(M_{Z}\right)=0.03374, \quad \alpha_{3}\left(M_{Z}\right)=0.1173
$$

and the SM beta functions, $b_{i}^{\mathrm{SM}}$ and $b_{i j}^{\mathrm{SM}}$ which read

$$
b_{i}^{\mathrm{SM}}=\left(\frac{41}{10},-\frac{19}{6},-7\right), \quad b_{i j}^{\mathrm{SM}}=\left(\begin{array}{ccc}
\frac{199}{50} & \frac{27}{10} & \frac{44}{5} \\
\frac{9}{10} & \frac{35}{6} & 12 \\
\frac{11}{10} & \frac{9}{2} & -26
\end{array}\right) .
$$

Open Access. This article is distributed under the terms of the Creative Commons Attribution License (CC-BY 4.0), which permits any use, distribution and reproduction in any medium, provided the original author(s) and source are credited.

\section{References}

[1] Planck collaboration, P.A.R. Ade et al., Planck 2015 results. XIII. Cosmological parameters, arXiv:1502.01589 [INSPIRE].

[2] Super-Kamiokande collaboration, Y. Fukuda et al., Evidence for oscillation of atmospheric neutrinos, Phys. Rev. Lett. 81 (1998) 1562 [hep-ex/9807003] [INSPIRE].

[3] SNO collaboration, Q.R. Ahmad et al., Direct evidence for neutrino flavor transformation from neutral current interactions in the Sudbury Neutrino Observatory, Phys. Rev. Lett. 89 (2002) 011301 [nucl-ex/0204008] [INSPIRE].

[4] KAmLAND collaboration, K. Eguchi et al., First results from KamLAND: evidence for reactor anti-neutrino disappearance, Phys. Rev. Lett. 90 (2003) 021802 [hep-ex/0212021] [INSPIRE].

[5] S. Davidson, E. Nardi and Y. Nir, Leptogenesis, Phys. Rept. 466 (2008) 105 [arXiv:0802.2962] [INSPIRE].

[6] C.S. Fong, E. Nardi and A. Riotto, Leptogenesis in the universe, Adv. High Energy Phys. 2012 (2012) 158303 [arXiv:1301.3062] [INSPIRE]. 
[7] T. Hambye, Leptogenesis: beyond the minimal type-I seesaw scenario, New J. Phys. 14 (2012) 125014 [arXiv:1212.2888] [INSPIRE].

[8] D. Aristizabal Sierra, J.F. Kamenik and M. Nemevšek, Implications of flavor dynamics for fermion triplet leptogenesis, JHEP 10 (2010) 036 [arXiv:1007.1907] [INSPIRE].

[9] D. Aristizabal Sierra, M. Dhen and T. Hambye, Scalar triplet flavored leptogenesis: a systematic approach, JCAP 08 (2014) 003 [arXiv: 1401.4347] [INSPIRE].

[10] ATLAS collaboration, Search for heavy neutrinos and right-handed $W$ bosons in events with two leptons and jets in pp collisions at $\sqrt{s}=7 \mathrm{TeV}$ with the ATLAS detector, Eur. Phys. J. C 72 (2012) 2056 [arXiv:1203.5420] [InSPIRE].

[11] CMS collaboration, Search for heavy neutrinos and $W$ bosons with right-handed couplings in proton-proton collisions at $\sqrt{s}=8 \mathrm{TeV}$, Eur. Phys. J. C 74 (2014) 3149 [arXiv:1407.3683] [INSPIRE].

[12] MEG collaboration, J. Adam et al., New constraint on the existence of the $\mu^{+} \rightarrow e^{+} \gamma$ decay, Phys. Rev. Lett. 110 (2013) 201801 [arXiv:1303.0754] [INSPIRE].

[13] Particle Data Group collaboration, K.A. Olive et al., Review of particle physics, Chin. Phys. C 38 (2014) 090001 [InSPIRE].

[14] G. Isidori, Y. Nir and G. Perez, Flavor physics constraints for physics beyond the standard model, Ann. Rev. Nucl. Part. Sci. 60 (2010) 355 [arXiv:1002.0900] [INSPIRE].

[15] G. D'Ambrosio, G.F. Giudice, G. Isidori and A. Strumia, Minimal flavor violation: an effective field theory approach, Nucl. Phys. B 645 (2002) 155 [hep-ph/0207036] [INSPIRE].

[16] R. Alonso, G. Isidori, L. Merlo, L.A. Muñoz and E. Nardi, Minimal flavour violation extensions of the seesaw, JHEP 06 (2011) 037 [arXiv:1103.5461] [INSPIRE].

[17] L. Di Luzio, R. Gröber, J.F. Kamenik and M. Nardecchia, Accidental matter at the LHC, JHEP 07 (2015) 074 [arXiv: 1504.00359] [INSPIRE].

[18] S.-Y. Ho, T. Toma and K. Tsumura, Systematic U(1) $B-L$ extensions of loop-induced neutrino mass models with dark matter, arXiv:1604.07894 [INSPIRE].

[19] M. Cirelli, N. Fornengo and A. Strumia, Minimal dark matter, Nucl. Phys. B 753 (2006) 178 [hep-ph/0512090] [INSPIRE].

[20] M. Cirelli, A. Strumia and M. Tamburini, Cosmology and astrophysics of minimal dark matter, Nucl. Phys. B 787 (2007) 152 [arXiv:0706.4071] [InSPIRE].

[21] M. Cirelli and A. Strumia, Minimal dark matter: model and results, New J. Phys. 11 (2009) 105005 [arXiv:0903.3381] [INSPIRE].

[22] D. Aristizabal Sierra, C. Simoes and D. Wegman, Closing in on minimal dark matter and radiative neutrino masses, JHEP 06 (2016) 108 [arXiv: 1603.04723] [INSPIRE].

[23] A. Ahriche, K.L. McDonald, S. Nasri and I. Picek, A critical analysis of one-loop neutrino mass models with minimal dark matter, Phys. Lett. B 757 (2016) 399 [arXiv:1603.01247] [INSPIRE].

[24] Y. Hamada, K. Kawana and K. Tsumura, Landau pole in the standard model with weakly interacting scalar fields, Phys. Lett. B 747 (2015) 238 [arXiv:1505.01721] [INSPIRE].

[25] R.N. Mohapatra and J.W.F. Valle, Neutrino mass and baryon number nonconservation in superstring models, Phys. Rev. D 34 (1986) 1642 [INSPIRE]. 
[26] M.B. Gavela, T. Hambye, D. Hernandez and P. Hernández, Minimal flavour seesaw models, JHEP 09 (2009) 038 [arXiv:0906.1461] [InSPIRE].

[27] D. Aristizabal Sierra, A. Degee and J.F. Kamenik, Minimal lepton flavor violating realizations of minimal seesaw models, JHEP 07 (2012) 135 [arXiv:1205.5547] [INSPIRE].

[28] P.S.B. Dev and A. Pilaftsis, Minimal radiative neutrino mass mechanism for inverse seesaw models, Phys. Rev. D 86 (2012) 113001 [arXiv:1209.4051] [INSPIRE].

[29] Mu3e collaboration, N. Berger, The Mu3e experiment, Nucl. Phys. Proc. Suppl. 248-250 (2014) 35 [INSPIRE].

[30] Mu3e collaboration, A. Bravar, The Mu3e experiment at PSI, Nucl. Part. Phys. Proc. 260 (2015) 155 [INSPIRE].

[31] Y. Kuno, PRISM/PRIME, Nucl. Phys. Proc. Suppl. 149 (2005) 376 [inSPIRE].

[32] R.J. Barlow, The PRISM/PRIME project, Nucl. Phys. Proc. Suppl. 218 (2011) 44 [InSPIRE].

[33] R. Alonso, M. Dhen, M.B. Gavela and T. Hambye, Muon conversion to electron in nuclei in type-I seesaw models, JHEP 01 (2013) 118 [arXiv:1209.2679] [INSPIRE].

[34] V. Cirigliano and M.J. Ramsey-Musolf, Low energy probes of physics beyond the standard model, Prog. Part. Nucl. Phys. 71 (2013) 2 [arXiv:1304.0017] [inSPIRE].

[35] S. Ando and K. Ishiwata, Constraints on decaying dark matter from the extragalactic gamma-ray background, JCAP 05 (2015) 024 [arXiv: 1502.02007] [INSPIRE].

[36] C. Rott, K. Kohri and S.C. Park, Superheavy dark matter and IceCube neutrino signals: bounds on decaying dark matter, Phys. Rev. D 92 (2015) 023529 [arXiv:1408.4575] [INSPIRE].

[37] A. Ibarra, A.S. Lamperstorfer and J. Silk, Dark matter annihilations and decays after the AMS-02 positron measurements, Phys. Rev. D 89 (2014) 063539 [arXiv:1309.2570] [INSPIRE].

[38] G. Giesen et al., AMS-02 antiprotons, at last! Secondary astrophysical component and immediate implications for dark matter, JCAP 09 (2015) 023 [arXiv: 1504.04276] [INSPIRE].

[39] E. Del Nobile, M. Nardecchia and P. Panci, Millicharge or decay: a critical take on minimal dark matter, JCAP 04 (2016) 048 [arXiv: 1512.05353] [INSPIRE].

[40] M. Cirelli, T. Hambye, P. Panci, F. Sala and M. Taoso, Gamma ray tests of minimal dark matter, JCAP 10 (2015) 026 [arXiv:1507.05519] [INSPIRE].

[41] C. Garcia-Cely, A. Ibarra, A.S. Lamperstorfer and M.H.G. Tytgat, Gamma-rays from heavy minimal dark matter, JCAP 10 (2015) 058 [arXiv:1507.05536] [INSPIRE].

[42] F. Capozzi, G.L. Fogli, E. Lisi, A. Marrone, D. Montanino and A. Palazzo, Status of three-neutrino oscillation parameters, circa 2013, Phys. Rev. D 89 (2014) 093018 [arXiv: 1312.2878] [INSPIRE].

[43] D.V. Forero, M. Tortola and J.W.F. Valle, Neutrino oscillations refitted, Phys. Rev. D 90 (2014) 093006 [arXiv: 1405.7540] [InSPIRE].

[44] M.C. Gonzalez-Garcia, M. Maltoni and T. Schwetz, Updated fit to three neutrino mixing: status of leptonic CP-violation, JHEP 11 (2014) 052 [arXiv:1409.5439] [INSPIRE].

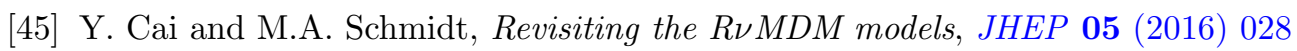
[arXiv: 1603.00255] [INSPIRE]. 
[46] J.F. Gunion, H.E. Haber, G.L. Kane and S. Dawson, The Higgs hunter's guide, Front. Phys. 80 (2000) 1 [INSPIRE].

[47] J. Hisano and K. Tsumura, Higgs boson mixes with an $\mathrm{SU}(2)$ septet representation, Phys. Rev. D 87 (2013) 053004 [arXiv: 1301.6455] [INSPIRE].

[48] M.J.G. Veltman, Limit on mass differences in the Weinberg model, Nucl. Phys. B 123 (1977) 89 [INSPIRE].

[49] R.H. Cyburt, J.R. Ellis, B.D. Fields and K.A. Olive, Updated nucleosynthesis constraints on unstable relic particles, Phys. Rev. D 67 (2003) 103521 [astro-ph/0211258] [INSPIRE].

[50] M. Kawasaki, K. Kohri and T. Moroi, Big-bang nucleosynthesis and hadronic decay of long-lived massive particles, Phys. Rev. D 71 (2005) 083502 [astro-ph/0408426] [INSPIRE].

[51] F. Bonnet, M. Hirsch, T. Ota and W. Winter, Systematic study of the D $=5$ Weinberg operator at one-loop order, JHEP 07 (2012) 153 [arXiv:1204.5862] [INSPIRE].

[52] D. Aristizabal Sierra, A. Degee, L. Dorame and M. Hirsch, Systematic classification of two-loop realizations of the Weinberg operator, JHEP 03 (2015) 040 [arXiv:1411.7038] [INSPIRE].

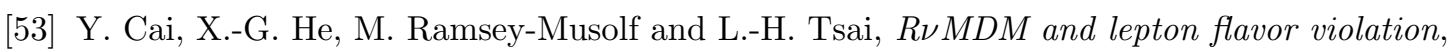
JHEP 12 (2011) 054 [arXiv:1108.0969] [INSPIRE].

[54] T.A. Chowdhury and S. Nasri, Lepton flavor violation in the inert scalar model with higher representations, JHEP 12 (2015) 040 [arXiv: 1506.00261] [INSPIRE].

[55] D. Aristizabal Sierra, Lepton flavor violation in radiative accidental matter models, in preparation.

[56] G. Passarino and M.J.G. Veltman, One loop corrections for $e^{+} e^{-}$annihilation into $\mu^{+} \mu^{-}$in the Weinberg model, Nucl. Phys. B 160 (1979) 151 [InSPIRE].

[57] J.A. Casas and A. Ibarra, Oscillating neutrinos and $\mu \rightarrow e, \gamma$, Nucl. Phys. B 618 (2001) 171 [hep-ph/0103065] [INSPIRE].

[58] A. Ibarra and G.G. Ross, Neutrino phenomenology: the case of two right-handed neutrinos, Phys. Lett. B 591 (2004) 285 [hep-ph/0312138] [INSPIRE].

[59] L. Lavoura, General formulae for $f_{1} \rightarrow f_{2} \gamma$, Eur. Phys. J. C 29 (2003) 191 [hep-ph/0302221] [INSPIRE].

[60] C.-H. Cheng, B. Echenard and D.G. Hitlin, The next generation of $\mu \rightarrow e \gamma$ and $\mu \rightarrow 3 e$ CLFV search experiments, in Community Summer Study 2013: Snowmass on the Mississippi (CSS2013), Minneapolis MN U.S.A. July 29-August 62013 [arXiv: 1309.7679] [INSPIRE].

[61] T. Aushev et al., Physics at super B factory, arXiv:1002.5012 [INSPIRE].

[62] D.R.T. Jones, The two loop $\beta$-function for a $G_{1} \times G_{2}$ gauge theory, Phys. Rev. D 25 (1982) 581 [INSPIRE].

[63] M.E. Machacek and M.T. Vaughn, Two loop renormalization group equations in a general quantum field theory. 1. Wave function renormalization, Nucl. Phys. B 222 (1983) 83 [INSPIRE]. 